This item was submitted to Loughborough's Research Repository by the author.

Items in Figshare are protected by copyright, with all rights reserved, unless otherwise indicated.

\title{
What would Wittgenstein say about social media?
}

PLEASE CITE THE PUBLISHED VERSION

https://doi.org/10.1177/1468794117713058

PUBLISHER

SAGE $\odot$ The Authors

VERSION

AM (Accepted Manuscript)

\section{PUBLISHER STATEMENT}

This work is made available according to the conditions of the Creative Commons Attribution-NonCommercialNoDerivatives 4.0 International (CC BY-NC-ND 4.0) licence. Full details of this licence are available at: https://creativecommons.org/licenses/by-nc-nd/4.0/

\section{LICENCE}

CC BY-NC-ND 4.0

\section{REPOSITORY RECORD}

Brooker, Phil, William Dutton, and Christian Greiffenhagen. 2019. "What Would Wittgenstein Say About Social Media?". figshare. https://hdl.handle.net/2134/25172. 


\title{
What Would Wittgenstein Say About Social Media?
}

Phil Brooker, William Dutton, and Christian Greiffenhagen

\begin{abstract}
Much of the excitement in social media analytics revolves around, a) capturing large-scale collections of naturally-occurring talk, b) repurposing them as data, and, c) finding ways to speak sociologically about them. Researchers have raised concerns over the use of social media data in research (eg boyd and Crawford, 2012; Housley et al, 2014; Tinati et al, 2014), exploring the ontological and epistemological grounding of the emerging field. We contribute to this debate by drawing on Wittgensteinian philosophy to elucidate hitherto neglected aspects; namely that it is not just social scientists who are in the business of analysing social media, but users themselves. We explore how mainstream social media analytics research (1) overinflates the importance of sociological theories, concepts and methodologies (which do not typically feature in the accounts of social media users), (2) downplays the extent to which social media platforms already exhibit order prior to any sociological accounting of them, and, (3) thereby produces findings which explain social scientific perspectives rather than the phenomena themselves. We reformulate the ontological and epistemological basis of social media analytics research from a Wittgensteinian perspective concerned with what it makes sense to say about social media, as members of society and as researchers studying those members. Such a project aims to explore social media users' language as a practice embedded within the context of social life and online communication. This reflects the everyday use of language as an evolving toolkit for undertaking social interaction, pointing towards an alternative conception of social media analytics.
\end{abstract}

Keywords: social media analytics; Big Data; ontology; epistemology; Wittgenstein; ordinary language philosophy; philosophy of social science. 


\section{What Would Wittgenstein Say About Social Media?}

Social media platforms are embedded in our cultural lives and are integral to how we think about social interaction. Because of this, and as part of a wider academic interest in 'Big Data', social researchers have increasingly engaged with social media platforms as vast collections of unscripted and unsolicited indicators of opinion and experience. Taking these collections as data has proven productive for researchers in the emerging field of social media analytics, who are increasingly able to use these data to explore issues pertinent to the traditional topical base of the social sciences (eg Baker and McEnery, 2015; Bingham-Hall and Law, 2015; Burnap et al, 2014; Currie et al, 2016). It has been to the field's credit that researchers have already sought to work through the conceptual and methodological implications social media analytics has brought in its wake. This is exemplified by recent work on an array of foundational concepts for Big Data research and social media analytics (eg boyd and Crawford, 2012; Housley et al, 2014; Tinati et al, 2014), where scholars seek to situate peoples' data generation and usage practices within the social sciences' theoretical, conceptual and methodological canon.

We contribute to this materializing body of work by drawing on the Ordinary Language Philosophy of Ludwig Wittgenstein $(1958,2009)$ to demonstrate different potential usages of social media data, and different questions to ask of them. This linkage between social media and Ordinary Language Philosophy belies an understanding of social media platforms as providing a (digitally-mediated) form of 'speaking'. Taking Twitter as an exemplar case (cf Tufekci, 2014), we might note that a range of language-usages - things that people do with language - are captured by the platform. These include telling stories, sharing information, promoting an agenda, joking, arguing, critiquing, reflecting, promising, positing, propositioning, defining, redefining, correcting, rebutting, disproving, praising, aggrandising, supporting, advertising, downplaying, reporting, retracting, apologising, justifying, summarising, detailing, generalising, stereotyping, and so on. Whilst not wishing to delimit a 
finite number of language-usages available to Twitter, this list serves as an example of how 'speaking' (as a term and practice) is made up of constituent practices. It is such practices that form the topic of Ordinary Language Philosophy's study (cf Coulter and Parsons (1990) on practices of 'seeing').

The keystone of our argument is thus. Social scientists are enthused by the analysis of social media, and especially so in deriving concepts, theories and methodologies to undertake the work. However, it is important also to recognise that social media users themselves are undertaking similar analytical work in the course of their social media usage - in order to understand and communicate with others, both researchers and social media users alike must constantly analyse, interpret and react to the interactional materials they are presented with (whether they be verbalised words and body gestures or the text and images that may be found within a tweet). This is how social life operates generally and ubiquitously, whether experienced offline or online ${ }^{1}$. It is equally important to recognise that social media users do all this analytic work without requiring a theory of their behaviour, without employing conceptual frameworks to support it, and without leveraging methodological principles to uncover those things ${ }^{2}$. Hence, whilst social media users are engaged in mutual understandings of how everyday life plays out in online spaces, social media analytics research is ostensibly about a different project; namely to incorporate social media usage into its own conceptual lexica, so as to be able to speak sociologically about what people do online, post-hoc. We counterpoint recent ('mainstream') ontological and epistemological work in social media analytics with a Wittgenstein-influenced take on social media, which is less concerned with applying social science theories, concepts and methods to social media and instead elects to describe the orders and organisation that already exist.

The paper proceeds as follows. We outline a key aspect of Wittgenstein's Ordinary Language Philosophy (OLP): his antipathy towards the social sciences as a means of capturing language- 
use and interaction to express things about the world, and his concentration on 'reminders' and 'perspicuous representations' as an alternative means to orient to these phenomena. We then summarise burgeoning ontological and epistemological work that seeks to lay the foundations for the ongoing development of social media analytics. Next, we evince OLP as an alternative to mainstream social media analytics ontological and epistemological thinking, arguing that by starting with a concern with the development and deployment of social science theories, concepts and methods mainstream approaches neglect to acknowledge the existing order and organisation that users bring to social media. As such, mainstream social science thereby produces findings which, we argue, herald insights primarily about their own approach than they do the phenomena they purport to explain. Though the approach is widespread throughout social media analytics, we demonstrate this by undertaking an OLP-oriented critique of two exemplar empirical studies of Twitter data (Heverin and Zach, 2012; Cha et al, 2010): first, in terms of how social science theory and concepts are leveraged to make (ontological) explanations of the world, and second, in terms of how those explanations are arrived at (epistemologically) through social science methods. This is followed by a more general discussion of OLP as a descriptive endeavour and the implications of this for mainstream social media analytics. We then return, in conclusion, to a Wittgensteinian concern with 'what it makes sense to say' about the multifarious usages of language-terms demonstrated through everyday social media usage. This, we posit, provides a different conceptualisation of social media analytics premised on descriptions of language-use as sets of practices embedded in social (and, in principle, online) contexts and which operate on the basis of a publically visible, witnessable and accountable order. Hence, the present paper contributes to social media analytics by exploring the potential for OLP to offer an innovative approach to the qualitative analysis of social media data.

\section{Wittgenstein's Ordinary Language Philosophy (In Brief) ${ }^{3}$}


Given that Wittgenstein scholars do not share a unified account of Wittgenstein's thought, producing a definite statement on what Wittgenstein's philosophy 'is about' is a somewhat perilous activity. Our reading will concentrate on the aspects of Wittgenstein's philosophy which justify a resistance against the appropriation of everyday language-use as materials by which to conceptualise social life.

In Philosophical Investigations (2009), Wittgenstein takes the academy to task on the idea of a purely referential language, and on the ontological and epistemological claims that result from such a view. A purely referential language is where the meanings of words are determined by defining them as associative with things in the world; objects, mental states, theoretical entities, etc. Under this view, how the word 'yellow' comes to mean anything is through pointing to yellow objects, requiring learners to associate the utterance of the word with some property of the object (eg the colour). Though Wittgenstein did not deny that language can be referential, he contended that philosophical mistakes arise from the idea of language as always and only garnered from referentiality as a fixed method for building and using language. As Hanfling (2006) articulates, Wittgenstein's rejection of an exclusively referential understanding of language is at least partially informed by a rejection of philosophy's tendency to conflate everyday language terms with philosophical concepts. As Wittgenstein notes:

\footnotetext{
'When philosophers use a word - "knowledge", "being”, "object", "I", "proposition/sentence", "name" - and try to grasp the essence of the thing, one must always ask oneself: is the word ever actually used in this way in the language in which it is at home?' (2009: §116)

'We [philosophers] take the possibility of comparison [between everyday and philosophical usages of words], which impresses us, as the perception of a highly general state of affairs.' (2009: §104)
} 
In this way, Wittgenstein argues that philosophers have become 'dazzled' $(2009, \S 100)$ by the answers that everyday usages of language appear to offer to philosophical problems. This compulsion may be encouraged by a cultural fascination with scientific and logical explanation:

'Philosophers constantly see the method of science before their eyes, and are irresistibly tempted to ask and to answer questions in the way science does. This tendency is the real source of metaphysics and leads philosophers into complete darkness.' (Wittgenstein, 1958: 18).

Hence, an understanding of the use of language becomes exasperated by certain activities associated with academic practice. For instance, solitary reflection on a word leads us to consider it in isolation, removed from a context where it is used unproblematically, and obscuring the standards surrounding its usage (Wittgenstein, 2009: §38, §593). In short, we philosophers and academics - become distracted by the meanings of words that are nearest to us in our own discipline-specific usages, forgetting that other different usages of the same word circulate elsewhere (ie in everyday life).

To guard against such confusion, Wittgenstein advocates observing how language is used in everyday situations:

'Consider, for example, the activities that we call "games". I mean boardgames, card-games, ball-games, athletic games, and so on. What is common to them all? - Don't say: “They must have something in common, or they would not be called 'games"" - but look and see whether there is anything common to all...Are they all 'entertaining'? Compare chess with noughts and crosses. Or is there always winning and losing, or competition between players? Think of patience.' (2009: §66) 
Hence, there are multiple different applications of the word 'game' which serve to resist a singular universal (ie scientific/logical) definition of what a game might be. Yet this does not upset how we deploy the term in our everyday life: as everyday people we know that professional football matches and rounds of Pac-Man are both legitimately describable as games. We are also unconcerned by the dissimilarities between the two activities which prevent the term 'game' from being a logical descriptor of a set of properties or qualities of games. Wittgenstein's OLP orients readers towards precisely these situations where word-use is unproblematic - the messy 'rough ground' (2009: §107) of everyday practices - to see how language functions in the context of its usage:

'It was correct that our considerations must not be scientific ones. The feeling 'that it is possible, contrary to our preconceived ideas, to think this or that' whatever that may mean - could be of no interest to us... And we may not advance any kind of theory. There must not be anything hypothetical in our considerations. All explanation must disappear, and description alone must take its place. And this description gets its light - that is to say, its purpose from the philosophical problems. These are, of course, not empirical problems; but they are solved through an insight into the workings of our language, and that in such a way that these workings are recognized - despite an urge to misunderstand them. The problems are solved, not by coming up with new discoveries, but by assembling what we have long been familiar with. Philosophy is a struggle against the bewitchment of our understanding by the resources of our language.' (2009: §109)

Hence, Wittgenstein proffers no theory of language, and rejects the possibility of such a theory at all. Instead, he elects to show readers different aspects of language in situ to elicit understandings of the various ways in which language gets used. Wittgenstein does so via 
'reminding' us (and encouraging us to remind ourselves) of the different usages of languageterms already in circulation, and by providing 'perspicuous representations' of those cases.

In our everyday language-use, we generally apply words correctly and unproblematically; as such, it can be illuminating to simply remind ourselves of the ways we use language terms, to recover the sense in which those terms have different uses in different contexts. Reminding is necessary, since we cannot give unified accounts of all applications and we need to 'see' them to understand the various manifestations:

'Something that one knows when nobody asks one, but no longer knows when one is asked to explain it, is something that has to be called to mind. (And it is obviously something which, for some reason, it is difficult to call to mind.)' (Wittgenstein, 2009: §91)

Building on this, Wittgenstein states a need to formulate these reminders as perspicuous representations, which should show examples of word use that are complete and at the same time open to review. It is the function of a language-term - what it is used to do - that makes a representation perspicuous, allowing us to compare and contrast the various uses a term may have. Working with language-terms in this way, we can isolate our deeply-held uses and see our motivation behind holding a certain view. We can ask: are we colouring particular examples of everyday language-use and social life with our philosophical/academic mindset, or are we describing them in such a way as to capture how they are embedded in the contexts in which they are given shape and meaning? This is how such examples provide perspicuous representation, and this is how perspicuous representations provide value in terms of understanding everyday life.

\section{Ontology, Epistemology, and the Foundations of Social Media Analytics}


It is apposite at this point to connect Wittgenstein's OLP with social media analytics, by exploring recent ontological and epistemological work in Big Data research. There have been concerns with the developmental direction of Big Data research and social media analytics since before research in the field had properly begun (see Savage and Burrows, 2007). These concerns have been formulated in a variety of studies seeking to work through the ontological and epistemological groundings of the field ${ }^{4}$. As Burrows and Savage note, the advent of Big Data research and social media analytics has made the time ripe to re-cover such questions: 'Big Data does challenge the predominant authority of sociologists and social scientists more generally to define the nature of social knowledge' (Burrows and Savage, 2014: 5). For these reasons, ontology and epistemology have emerged as critical debates for the field.

Ontological and epistemological ${ }^{5}$ studies within Big Data research and social media analytics represent an array of approaches, yet nonetheless display shared motivations around: a) capturing collections of naturally-occurring talk, opinions, attitudes and experiences on social media platforms, b) repurposing these collections as data to derive empirical insights, and c) finding ways to interpret those data by connecting them to existing social science concepts and theories. Throughout all three of these stages in the wider social media analytics strategy, ontological and epistemological thinking is evident.

For instance, of the potential for using social media to capture collections of naturallyoccurring talk, opinions, attitudes and experiences, Housley et al note that 'these technologies and their allied data have the potential to "digitally-remaster" classic questions about social organization, social change and the derivation of identity from collective life.' (2014: 4). These collections, however, are not to be taken uncritically, and it is important to use data as a way to access the ontological and epistemological assumptions we are making when we draw them from their native environments. As Felt reminds us: 
'It is not enough to simply imagine social media posts as a "raw" artifact, separated from the systems that produced them. Researchers must consider the processes that produced them. Researchers must consider the processes that create a moment as well as the networks involved in the situation. This must extend to the algorithms utilized to present the data.' (Felt, 2016: 6)

Following data collection through to empirical insights requires an intermediary stage where methods and methodologies can be leveraged to realise collections of social media posts as research data. This process too has encapsulated various ontological and epistemological ideas for researchers. For instance, Edwards et al note that 'Rather than regarding social media data analysis as a surrogate for traditional methods... the experience of digital social research to date suggests the need for an account of how it might augment, and be augmented by, traditional social research methods.' (2013: 247). Rogers highlights the notion of 'repurposing' as particularly important here:

'Digital methods repurpose or build on top of the dominant devices of the medium, and in doing so make derivative works from the results, figuratively and literally. That is, the initial outputs may be the same as or similar to those from online devices, but they are seen or rendered in new light, turning what was once familiar - a page of engine results, a list of tweets in reverse chronological order, a collection of comments, or a set of interests from a social networking profile - into indicators and findings.' (Rogers, 2013: 3)

Researchers have also sought to connect such indicators and findings with existing social theories and concepts, thereby lending wider relevance to their empirical work as part of the broader social science research canon. As Kitchin states, 'It is one thing to identify patterns; it is another to explain them. This requires social theory and deep contextual knowledge' (2014: 
8), and it has been argued that only 'once these [empirical] advances have become more embedded and consolidated within theoretical frames' (Cowls and Schroeder, 2015: 3) can social media analytics push social science into new domains. Murthy states exactly these aims at the outset of his recent work on Twitter:

'Although I examine the practice of social media through specific Twittermediated events, this book's emphasis is both explanatory and theoretical. Specifically, my prime aim is to better understand the meanings behind Twitter and similar social media through concise yet sophisticated interpretations of theories of media and communication' (Murthy, 2013: xii)

More recently, Lupton and others (eg Beer, 2016; Boellstorff, 2016) have sought to use arguments drawn from Science and Technology Studies to 'identify and think through some of the ways in which sociocultural theory may contribute to understanding data practices' (2016: 1). In this way, social media analytics is integrating ever more closely with the wider sociological project within which it originates, by way of exploring how other sociological subdisciplines with similarly pertinent ontological and epistemological concerns have embraced those issues.

\section{An Ordinary Language Philosophy Take on Social Media Analytics}

Above, we have characterised social media analytics as, amongst other things, seeking to methodologically and epistemologically repurpose the naturally-occurring collections of online talk as data (cf Rogers, 2013) and to produce ontological claims about those collections by mapping them onto social science theories (cf Beer, 2016; Boellstorff, 2016; Cowls and Schroeder, 2015; Kitchin, 2014; Lupton, 2016). Yet seemingly despite the renewed focus on ontology and epistemology, these treatments of data simply demonstrate that it is possible to talk about social media with sociological terminology without providing a justification as to 
what the value in doing so might be. Hence, with OLP in mind, we more carefully unpick what such studies claim of their data. Provocatively, we suggest there are grounds to read mainstream social media analytics studies as failing to account for their chosen subjects in a way that increases our understanding of those subjects.

To concretise the concerns of OLP as an alternative to mainstream social media analytics research, we now present two cases of empirical studies of Twitter data to locate their respective mis-steps: one ontological (Heverin and Zach, 2012) and one epistemological (Cha et al, 2010). To illustrate our concerns we will focus on each of these two particular studies as exemplar cases, followed by brief descriptions of other studies where similar ontological or epistemological mistakes are evident to show how the problems pervade more generally across social media analytics. Our concern is not with the studies themselves, but their capacities as representative of the ontological and epistemological confusions that arise from mainstream social media analytics studies ${ }^{6}$.

\section{Ontology: A Mis-Step in the Use of Theory and Concepts}

Heverin and Zach (2012) study the usage of Twitter hashtags as a communication medium around three U.S. campus shootings. They describe the various different sorts of interactional work undertaken by users at different points along the course of each event:

'Through an analysis of the content of the microblogging communications sent via Twitter during three similar, violent crises, we found that information-sharing behaviors dominated the early response phase, also known as the critical period, of violent crises. We also found that opinion sharing increased considerably during the critical period and peaked in all three crises during the recovery phase.' (2012: 34) 
To situate these descriptions within the wider social science literary canon, Heverin and Zach draw on Dervin's (1983) theory of collective sense-making, 'defined as a process by which individuals attempt to bridge the cognitive gaps that they face in everyday life as well as during nonroutine times such as crises' (2012: 34). For Heverin and Zach, the concept of sense-making explains the communicative practices described as an ontological claim about the world:

'Frameworks such as Dervin's (1983) sense-making theory help provide a basis for understanding how individual behaviors can contribute to the sensemaking of others and expand the way in which we can look at microblogging communications by applying [a] user-centred approach to understanding information needs during crisis situations from the perspective of the individual actors' (2012: 45)

From the OLP perspective, the descriptive element of Heverin and Zach's (2012) study - the investigation of how language is used in specific settings to do certain types of work - is where its value lies. However, Heverin and Zach make an ontological leap in their claim that the communicative practices they investigate constitute a demonstration of a theoretical construct (ie collective sense-making). Given this, it is a more compelling account of Heverin and Zach's (2012) study that the conceptual orientation to sense-making is less explanatory of the communicative practices to which it is ostensibly applied, and more explanatory of the social scientific enterprise generally in that it demonstrates a commitment to producing concepts around the practices social scientists are in the business of observing.

Similar ontological leaps are made by Cheung and To (2016) in their characterising of the social media interactions between consumers and corporations on social media via the theory of planned behaviour, by Ellison et al in their examination of Facebook users' practices of mobilising help from their networks via "social capital conversions" (2013: 155) and by 
Tufekci's (2008) investigation of privacy on Facebook and Myspace through the lens of Altman's theory of privacy as a process of optimization between self-disclosure and withdrawal. In all of these cases, it is unclear as to what subsequently explaining users' practices in terms of their respective a priori theoretical commitments (and thereby abstracting the explanatory contribution of the study beyond the user-practices they purport to study) adds to our understanding of them.

\section{Epistemology: A Mis-Step in the Use of Methods and Methodologies}

Cha et al (2010) investigate the notion of 'influence' on Twitter - how it is exerted and responded to by others. They begin by noting Twitter as an area where our existing conceptualisation of influence does not apply neatly:

'The traditional influentials theory [where one person has influence and others connected to them are thereby influenced] has...been criticised because its information flow process does not take into account the role of ordinary users' (2010: 11)

Here, ordinary users are seen as having greater agency in exerting and responding to influence than in traditional models, which the authors deem more befitting the 'viral' nature of Twitter. To enable their investigation into influence, Cha et al (2010) make an epistemological leap to render peoples' orientations to influence amenable to social scientific analysis. This is performed by the 'datafication' of influence into 'three "interpersonal" activities on Twitter" ... [which] represent the different types of influence of a person' (Cha et al, 2010: 11-12), to be measured and explained via statistical analysis.

The initial premise - Cha et al's (2010) orientation to influence as consisting of communicative practices demonstrable within users tweets - is appealing. Descriptions of the ways in which 
influence was performed and achieved by tweeters in practice would be highly illuminating. Yet it is the subsequent (epistemological) leap that we find problematic from the OLP perspective. In order to explore influence as a phenomenon, Cha et al (2010) reduce influence down to the three aforementioned metadata elements (ie followers, retweets and mentions). Hence, the study produces a statistical analysis of how these three modes of interaction correlate with one another, with findings indicating such things as tweeters who receive more retweets and mentions are more influential than those who simply have a higher number of followers. However, the repurposing of interactions into statistical variables taken to stand for 'influence' is an epistemological move that fails to increase our understanding of what influence is to the tweeters who exert and respond to it in the context of their everyday Twitter lives. Cha et al's (2010) study promises an exploration of how influence is experienced by Twitter users, yet their epistemological work undermines the extent to which the study can capture users experiences of influence as being expressed through the language and contextual background of a tweet. Hence, we might rather see the analyses offered by Cha et al (2010) as being less about 'influence' and more a demonstration of 'datafication' as a method of producing analyses of large volume social media data that accord with the traditional social science methodological toolkit.

Such a move is apparent also in the work of Ceron et al (2014) who model political preference on Twitter with a semi-automated version of sentiment analytic metrics for linguistically coding large data corpora, in Huang et al's (2013) characterisation of 'online proximity' (ie the feeling of closeness or distance from those we interact with online) as being measurable through space, time and homophily, and in Gilbert and Karahalios' development of "tie strength" (2009: 212) as a means of using various interactions and metadata to measure relationships between Facebook users. However, as with Cha et al's (2010) study, it is unclear as to what these 
'datafied' analyses of digitally-mediated interactions might relate to and/or represent the phenomena they purport to be about.

\section{Discussion: Explanation and Description}

Given the negative problematising that OLP applies to mainstream social media analytics, it is important also to explore how OLP might be used positively in terms of providing researchers with an alternative entry point into social media. Here, we build on OLP's affinity towards description (as opposed to explanation) as a means of understanding social life. The questions here are: what counts as an explanation of the things we (social media analysts) seek to understand about the world? And how can we access those explanations? As we have argued, social media analytics is currently exploring answers to these questions via ontological and epistemological moves. However, if our goal is to understand peoples' practices when they use Twitter to communicate within specific contexts, then OLP reminds us that we would do well to concentrate, foremost, on producing descriptions of those activities. ${ }^{8}$ As Winch - an advocate of Wittgenstein's OLP - suggests, description can provide an equally illuminating alternative to theoretical and conceptual explanation:

'Understanding is the goal of explanation and the end-product of successful explanation. But of course it does not follow that there is understanding only where there has been explanation; neither is this in fact true.' (1990: $\mathrm{x})$

This focus on description attunes us to peoples' own accounts of the activities they are undertaking, and encourages us to think with clarity on how theirs and our descriptions do the work of describing (ie how we might recognise one activity as a particular kind of activity and not something else). Notably, it is a rare case where social theories and concepts might be found within everyday language use - as Hutchinson et al note: 
'It is not, first, for sociologists to decide what someone is properly said to be doing. The language they are using, after all, does not belong to them, but is one that they speak because they belong to the language communities about and within which they write: 'washing hands' isn't a description that any sociologist has contrived, and it is indisputable that whether it is the correct thing to say or not should be decided by the way in which language works, is used, within the activities to which it belongs (to domestic affairs: clean those dirty hands!; to medical situations: 'scrub up before surgery'; or to affairs of state: washing hands as the ceremonial way to rescue oneself).' (2008: 77-78)

Thus, to simply say that someone is 'washing their hands' without a sense of the context surrounding the statement provides only an incomplete description which immediately begs further questions - who is washing their hands, and why? It is precisely such context that lends any description of a given activity its intelligibility and its criteria for correct application. And it is the visibility of those contexts and criteria within language-use (eg using the term 'scrubbing up' as a distinctively medicalised turn of phrase rather than just 'washing my hands') and social interaction (eg wearing green overalls and working in a hospital) which provide ample footing for researchers seeking to understand how social life is organised.

Though not necessarily within the topical remit of social media analytics, several researchers in the fields of Human-Computer Interaction and Computer-Supported Cooperative Work have undertaken qualitative analyses that do attend to the everyday practices through which people generate and use digital data (eg Didžiokaitè et al, 2017; Brown et al, 2013; Brown et al, 2015; Licoppe et al, 2016; Reeves and Brown, 2016; Reeves et al, 2015; Rost et al, 2013) and these works provide insight into how we might put Wittgenstein's ideas to practice. For instance, Rost et al (2013) examine users' 'check-in' practices on Foursquare, Brown et al (2015) explore mobile internet searches as an activity jointly organised around information retrieval and 
conversational interaction, and Licoppe et al (2016) analyse the conversational and interactional practices through which users establish matches on the gay dating app Grindr. All of these studies are premised, first and foremost, on deep descriptions of the interactions at hand. What these studies demonstrate is an attunement to the practical interactions that play out through and are afforded by digital platforms, as opposed to extracting those interactions out of their local contexts via 'datafying' them or analysing them according to their fit with sociological theories or concepts.

Turning back now to social media analytics, we can see that researchers in this areas do not typically share this focus on producing descriptions grounded in the interactional contexts available through social media data, and this has led to ontological and epistemological missteps. Taking our exemplar cases for instance, both Heverin and Zach (2012) and Cha et al (2010) allude to the possibility of describing the activities they take as their subjects in such a way, yet neither focus on description as the primary purpose of the study. Heverin and Zach (2012) downplay the descriptive element of tweeting practices around campus shooting events in favour of exploring how those activities might be explained with reference to "collective sense-making”. Cha et al (2010) set up the notion of influence in such a way as to make a descriptive account possible, yet neglect to undertake the descriptive work in favour of a 'datafied' version of influence based on a small selection of tweeting practices. This connects back to Wittgenstein's comments, outlined above, on language as not purely referential: the aforementioned researchers commit referential fallacies when they claim that social media users' interactions map neatly onto the existing theory of collective-sense making (Heverin and Zach, 2012), and when they work from the assumption that social media users' conception of 'influence' is identical to the definition of 'influence' in social science research and has the capacity to be neatly encapsulated in a simplistic model based on the 'datafication' of three metrics (Cha et al, 2010). In making these referential fallacies, both studies produce 
explanations, yet the ontological and epistemological work involved in producing those explanations gives grounds to question whether what they are explaining is the subjects they profess to study or the assumptions and methods that social media analytics employs to make sense of the world.

As Wittgenstein might have it, both of these studies (and those listed alongside them) are dazzled by the idea that the everyday language captured in social media data can be used to produce direct answers to social scientific problems. However, social media analytics typically operates without acknowledging the effects of the translation of social media posts from 'everyday language' to 'data' and 'analyses', ie where we typically undertake ontological and epistemological work to wrangle the phenomena at hand into shapes we can comfortably handle. This has been, in fact, a lingering promise of 'Big Data' research (as the substrate of social media analytics) for academia as well as in marketing, industry, policy and other sectors working in the emerging field: that new data sources and new computational techniques can afford quick, cheap, highly-reproducible, impactful, theoretically-informative and methodologically-innovative empirical findings (cf Savage and Burrows, 2007, 2009; Burrows and Savage, 2014). Social scientists, with the keen methodological focus that characterises their field, have been amongst the most critical and reflexive users of social media data (eg boyd and Crawford, 2012; Housley et al, 2014; Tinati et al, 2014) and this is to their credit. However, Wittgenstein's OLP - chiefly, his antipathy towards the academy's objectifying of everyday language - provides ample justification for us to have to reconsider how we use social media data in our attempts to understand the lives of those that generate them. We argue that this is linked to notion that the social sciences (and by extension, social media analytics) consist of 'second-order' disciplines which are inevitably one-step removed from the phenomena they take as their subject (cf Schutz, 1972). For example, in producing these kinds of (second-order) study and in acceding to the dream-myth of objective (social) science that Wittgenstein warns 
against, both Heverin and Zach (2012) and Cha et al (2010) miss an opportunity to provide deep descriptions of the activities and phenomena they purport to investigate. Such descriptions might provide a means of reorienting to - being 'reminded' of with perspicuous representations - how people use language to conduct their social activities on platforms such as Twitter. It is precisely these kinds of descriptions that are lacking amongst social media analytics, and which Wittgenstein's OLP encourages us to strive for.

\section{Concluding Remarks}

Throughout this paper we have elected to make an analogy between the current social media analytics zeitgeist and the ontological and epistemological approach of the social sciences that Wittgenstein's OLP situated itself against. We have used this analogy to argue that inasmuch as social media analytics provides a motivation to revisit the ontological and epistemological foundations of social research, we should also not neglect the opportunity to revisit those bodies of work (eg OLP) that critiqued and problematised those foundations first time around. We do not claim that it is mistaken to focus on ontology and epistemology as key concerns of the developing field - the theoretical/conceptual explanations and methodological approaches of the social sciences (enabled by a concern with ontology and epistemology) are incomparably adept at such things as motivating social change and activism, providing counter-narratives, facilitating critical reflection, and so on. Rather, our two-fold claim is thus. Firstly, it is wrongfooted to operate as if the particular ontological and epistemological perspectives evident in mainstream social media analytics research can provide direct insight into 'reality' as it is often assumed they do, and researchers must be careful to explore where their insights originate from and accordingly what they can be said to reflect. Secondly, OLP stands as a particularly antagonistic alternative/non-mainstream approach to social science which has received little attention, yet which is worthy of further exploration. 
What exactly an OLP approach to social media research might look like 'on the shop floor' is difficult to envisage, given the idiosyncrasies which can be expected to arise in empirical studies of this ilk (ie where the interactional context is everything). We have however briefly highlighted the works of several researchers (Didžiokaitè et al, 2017; Brown et al, 2013; Brown et al, 2015; Licoppe et al, 2016; Reeves and Brown, 2016; Reeves et al, 2015; Rost et al, 2013) who attend specifically to the interactional contexts within which digitally-mediated social interactions take place. In applying these kinds of ideas to social media analytics specifically, what we hope to have established throughout this paper is a sense of the value and the requirements of an OLP alternative to mainstream social media analytics, as well as pointing the way towards strategies for conducting research in this way. The strategies we hint at are intended to encourage researchers to maintain an orientation to what it makes sense to say about and with social media, and to seek out the methods by which social media users publicly display their sense-making processes through their language-in-context. It is in these ways that the order and organisation that is essential to social media (as a form of communicative languageuse) becomes available to researchers. Describing this order and organisation has the potential to give social media research deep insight into what people use language-on-social-media to do in their lives.

As a final point: we are reluctant to speak on behalf of Wittgenstein in terms of providing an answer to the title of the paper - we can only speculate what he might have had to say about social media. Nonetheless, should Wittgenstein have had anything to say at all about the subject, we hope that we have reflected how he might have said it in such a way as to allow social media researchers to take up the mantle for themselves. 


\section{Endnotes}

1. This focus on the organisation of social life through everyday interactions is made particularly tangible by ethnomethodology (cf Garfinkel, 1967, 2002); itself a field strongly influenced by Wittgensteinian philosophy.

2. We note a parallel between Ordinary Language Philosophy's motivations to resist the 'scientific' appropriation of everyday language usage and Schutz' (1972) conception of the social sciences as a 'second-order' discipline. We return to this idea briefly in later sections.

3. Wittgenstein's thinking is split between pre- and post-Tractatus ([1922] 2005) periods, reflecting a significant change of direction in his philosophy. We will be drawing on Wittgenstein's post-Tractatus philosophy, especially that exhibited in his Philosophical Investigations ([1953] 2009).

4. Such works include: Beer, 2016; Beer and Burrows, 2013; Boellstorff, 2016; Brooker et al, 2016a; Brooker et al, 2016b; Burrows and Savage, 2014; Cowls and Schroeder, 2015; Edwards et al, 2013; Felt, 2016; Gillespie, 2014; Housley et al, 2014; Kennedy, Poell and van Dijck, 2015; Kitchin, 2014; Kitchin and McArdle, 2016; Lupton, 2016; Marshall, 2012; Murthy, 2013; Rogers, 2013; Savage and Burrows, 2009; Tinati et al, 2014; Tufekci, 2014; van Dijck, 2013; Vis, 2013.

5. Although the two terms are inevitably interrelated, we use the term ontology to refer to the study of the nature of reality and epistemology to refer to our methods of finding out about reality. The distinction is between 'What kinds of thing really exist in the world?' (Hughes and Sharrock, 1997: 5) as an ontological question and 'How is it possible, if it is, for us to gain knowledge of the world?' (Hughes and Sharrock, 1997: 5) as its epistemological counterpart. 
6. Other such studies include: Baker and McEnery, 2015; Bingham-Hall and Law, 2015; Bronson and Knezevic, 2016; Burnap et al, 2014; Christiaens, 2016; Citron, 2014; Currie et al, 2016; Lachlan et al, 2014; Markham and Baym, 2009; Vidal et al, 2015; and many more.

7. These are: 1) the number of followers a user has; 2) the number of retweets their posts garner, and; 3) the frequency with which they are mentioned by other users.

8. It is worth noting that similar critiques have been levied at other areas of social science and the social scientific enterprise more generally (eg Button et al, 1995; Button and Sharrock, 1993; Randall and Sharrock, 2011; Sharrock and Anderson 1984). Our focus on social media analytics specifically is motivated by our seeing these issues occurring again in the newlyemerging field.

\section{Declaration of Conflicting Interests}

The authors declare that there is no conflict of interest. 


\section{References}

Baker P and McEnery T (2015) Who benefits when discourse gets democratised? Analysing a Twitter corpus around the British Benefits Street debate. In: Baker P and McEnery T (eds) Corpora and Discourse Studies: Integrating Discourse and Corpora. Basingstoke: Palgrave Macmillan, pp.244-265.

Beer D (2016) How should we do the history of Big Data?. Big Data and Society 3(1): 1-10.

Beer D and Burrows R (2013) Popular culture, digital archives and the new social life of data. Theory, Culture and Society 30(4): 47-71.

Bingham-Hall J and Law S (2015) Connected or informed?: Local Twitter networking in a London neighbourhood. Big Data and Society 2(2): 1-17.

Boellstorff T (2016) For whom the ontology turns: Theorizing the digital real. Current Anthropology 57(4): 387-407.

boyd d and Crawford K (2012) Critical questions for Big Data: Provocations for a cultural, technological, and scholarly phenomenon. Information, Communication and Society 15(5): 662-679.

Bronson K and Knezevic I (2016) Big Data in food and agriculture. Big Data and Society 3(1): $1-5$.

Brooker P, Barnett J and Cribbin T (2016a) Doing social media analytics. Big Data \& Society 3(2): $1-12$.

Brooker P, Barnett J, Cribbin T and Sharma S (2016b) Have we even solved the first 'Big Data challenge'? Practical issues concerning data collection and visual representation for social media analytics. In: Snee H, Hine C, Morey Y, Roberts S and Watson H (eds) Digital Methods for Social Science: An Interdisciplinary Guide to Research Innovation. Basingstoke, UK: Palgrave Macmillan, pp.34-50. 
Brown B, McGregor M and Laurier E (2013) iPhone in vivo: Video analysis of mobile device use. In: Proceedings of the SIGCHI Conference on Human Factors in Computing Systems (CHI '13). Paris, France, 27 April-2 May 2013, pp.1031-1040. New York, USA: ACM.

Brown B, McGregor M and McMillan D (2015) Searchable objects: Search in everyday conversation. In: Proceedings of the $18^{\text {th }}$ ACM Conference on Computer Supported Cooperative Work \& Social Computing (CSCW '15). Vancouver, BC, Canada, 14-18 March 2015, pp.508-517. New York, USA: ACM.

Burnap P, Williams ML, Sloan L, Rana O, Housley W, Edwards A, Procter R and Voss A (2014) Tweeting the terror: Modelling the social media reaction to the Woolwich terrorist attach. Social Network Analysis and Mining 4(1): 1-14.

Burrows R and Savage M (2014) After the crisis? Big Data and the methodological challenges of empirical sociology. Big Data and Society 1(1): 1-6.

Button G, Coulter J, Lee JRE and Sharrock W (1995) Computers, Minds and Conduct. Cambridge: Polity Press.

Button G and Sharrock W (1993) A disagreement over agreement and consensus in constructionist sociology. Journal for the Theory of Social Behaviour 23(1): 1-25.

Ceron A, Curini L, Iacus SM and Porro G (2014) Every tweet counts? How sentiment analysis of social media can improve our knowledge of citizens' political preferences with an application to Italy and France. New Media \& Society 16(2): 340-358.

Cha M, Haddadi H, Benevenuto F and Gummadi KP (2010) Measuring user influence in Twitter: The million follower fallacy. In: Proceedings of the Fourth International AAAI Conference on Weblogs and Social Media (ICWSM '10), Washington, USA, 23-26 May 2010, pp.10-17. Menlo Park, California: The AAAI Press. 
Cheung MFY and To WM (2016) Service co-creation in social media: An extension of the theory of planned behaviour. Computers in Human Behavior 65: 260-266.

Citron DK (2014) Hate Crimes in Cyberspace. London: Harvard University Press.

Cowls J and Schroeder R (2015) Causation, correlation, and Big Data in social science research. Policy and Internet 7(4): 447-472.

Coulter J and Parsons ED (1990) The praxiology of perception: Visual orientations and practical action. Inquiry 33: 251-272.

Currie M, Paris BS, Pasquetto I and Pierre J (2016) The conundrum of police officer-involved homicides: Counter-data in Los Angeles County. Big Data and Society 3(2): 1-14.

Dervin B (1983) An overview of sense-making research: Concepts, methods, and results to date, In: Annual Meeting of the International Communications Association, Dallas, Texas.

Didžiokaitė G, Saukko P and Greiffenhagen C (2017) The mundane experience of everyday calorie trackers: Beyond the metaphor of Quantified Self. New Media \& Society [online first]: $1-18$.

Edwards A, Housley W, Williams M, Sloan L and Williams M (2013) Digital social research, social media and the sociological imagination: Surrogacy, augmentation and reorientation. International Journal of Social Research Methodology 16(3): 245-260.

Ellison NB, Gray R, Vitak J, Lampe C and Fiore AT (2013) Calling all Facebook friends: Exploring requests for help on Facebook. In: Proceedings of the Seventh International AAAI Conference on Weblogs and Social Media (ICWSM '17). Ann Arbor, MI, USA, 8-11 July 2013, pp.155-164. Washington, DC, USA: AAAI.

Felt M (2016) Social media and the social sciences: How researchers employ Big Data analytics. Big Data and Society 3(1): 1-15.

Garfinkel H (1967) Studies in Ethnomethodology. New Jersey: Prentice Hall, Inc. 
Garfinkel H (2002) Ethnomethodology’s Program: Working Out Durkheim's Aphorism (edited and introduced by Warfield Rawls A). Oxford: Rowman and Littlefield Publishers, Inc. Gilbert E and Karahalios K (2009) Predicting tie strength with social media. In: Proceedings of the SIGCHI Conference on Human Factors in Computing Systems (CHI ‘09). Boston, MA, USA, 4-9 April 2009, pp.211-220. New York, USA: ACM.

Gillespie T (2014) The relevance of algorithms. In Gillespie T, Boczkowski PJ and Foot KA (eds) Media Technologies: Essays on Communication, Materiality, and Society. Cambridge, MA: The MIT Press, pp.167-193.

Hanfling O (2006) Wittgenstein and the Human Form of Life. London: Routledge.

Heverin T and Zach L (2012) Use of microblogging for collective sense-making during violent crises: A study of three campus shootings. Journal of the American Society for Information Science and Technology. 63(1): 34-47.

Housley W, Procter R, Edwards A, Burnap P, Williams M, Sloan L, Rana O, Morgan J, Voss A and Greenhill A (2014) Big and broad social data and the sociological imagination: A collaborative response. Big Data and Society 1(2): 1-15.

Huang Y, Shen C and Contractor NS (2013) Distance matters: Exploring proximity and homophily in virtual world networks. Decision Support Systems 55: 969-977.

Hughes JA and Sharrock WW (1997) The Philosophy of Social Research. Essex: Pearson Education Limited.

Hutchinson P, Read R and Sharrock WW (2008) There is No Such Thing as a Social Science: In Defence of Peter Winch. Hampshire: Ashgate Publishing Limited.

Kennedy H, Poell T and van Dijck J (2015) Data and agency. Big Data and Society 2(2): 1-7. Kitchin R (2014) Big Data, new epistemologies and paradigm shifts. Big Data and Society 1(1): 1-12. 
Kitchin R and McArdle G (2016) What makes Big Data, Big Data? Exploring the ontological characteristics of 26 datasets. Big Data and Society 3(1): 1-10.

Lachlan K, Spence PR and Lin X (2014) Expressions of risk awareness and concern through Twitter: On the utility of using the medium as an indication of audience needs. Computers in Human Behavior 35: 554-559.

Licoppe C, Rivière CA and Morel J (2016) Grindr casual hook-ups as interactional achievements. New Media \& Society 18(1): 2540-2558.

Lupton D (2016) Digital companion species and eating data: Implications for theorising digital data-human assemblages. Big Data and Society 3(1): 1-5.

Markham AN and Baym NK (eds) (2009) Internet Inquiry: Conversations About Method. London: Sage.

Marshall C (2012) Big Data, the crowd and me. Information Services and Use 32: 215-226.

Murthy D (2013) Twitter: Social Communication in the Twitter Age. Cambridge: Polity Press.

Randall D and Sharrock W (2011) The sociologist as movie critic. In: Rouncefield M and Tolmie P (eds) Ethnomethodology at Work. Farnham, Surrey: Ashgate Publishing Ltd, pp.1-18.

Reeves S and Brown B (2016) Embeddedness and sequentiality in social media. In: Proceedings of the $19^{\text {th }}$ ACM Conference on Computer-Supported Cooperative Work \& Social Computing (CSCW '16). San Francisco, California, USA, 27 February-2 March 2016, pp.1052-1064. New York, USA: ACM.

Reeves S, Martindale S, Tennent P, Benford S, Marshall J and Walker B (2015) The challenges of using biodata in promotional filmmaking, ACM Transactions on Computer-Human Interaction (TOCHI) 22(3), 11.

Rogers R (2013) Digital Methods. London: The MIT Press. 
Rost M, Barkhuus L, Cramer H and Brown B (2013) Representation and communication: Challenged in interpreting large scale social media datasets. In: Proceedings of the 2013 Conference on Computer Supported Cooperative Work (CSCW '13). San Antonio, Texas, USA, 23-27 February 2013, pp.357-362. New York, USA: ACM.

Savage M and Burrows R (2007) The coming crisis of empirical sociology, Sociology 41(5): 885-899.

Savage M and Burrows R (2009) Some further reflections on the coming crisis of empirical sociology. Sociology 43(4): 762-777.

Schutz A (1972) The Phenomenology of the Social World. USA: Northwestern University Press.

Sharrock WW and Anderson RJ (1984) The Wittgenstein connection. Human Studies 7: 375386.

Tinati R, Halford S, Carr L and Pope C (2014) Big Data: Methodological challenges and approaches for sociological analysis. Sociology 48(4): 663-681.

Tufekci Z (2008) Can you see me now? Audience and disclosure regulation in online social network sites. Bulletin of Science, Technology \& Society 28(1): 20-36.

Tufekci Z (2014) Big questions for social media big data: Representativeness, validity and other methodological pitfalls. In: Proceedings of the Eighth International AAAI Conference on Weblogs and Social Media, Ann Arbor, Michigan, USA, 1-4 June 2014, pp.505-514. Palo Alto, California, USA: The AAAI Press.

van Dijck J (2013) The Culture of Connectivity: A Critical History of Social Media. Oxford: Oxford University Press.

Vidal L, Ares G, Machin L and Jaeger S (2015) Using Twitter data for food-related consumer research: A case study on 'what people way when tweeting about different eating situations'. Food Quality and Preference 45: 58-69. 
Vis F (2013) A critical reflection on Big Data: Considering APIs, researchers and tools as data makers. First Monday, 7 October 2013. Available at: http://ojs-prodlib.cc.uic.edu/ojs/index.php/fm/article/view/4878/3755 (accessed 4 August 2016).

Winch P (1990) The Idea of a Social Science, and Its Relation to Philosophy. London: Routledge and Kegan Paul.

Wittgenstein L (1958) The Blue and Brown Books. Oxford: Blackwell.

Wittgenstein L (2005) Tractatus Logico-Philosophicus (translated by Ogden CK). Abingdon, UK: Routledge.

Wittgenstein L (2009) Philosophical Investigations (translated by Anscombe GEM, Hacker PMS and Schulte J). Chichester, UK: Wiley-Blackwell. 